\title{
Performance of the Abbott Real Time CT/NG assay in urines and cervico- vaginal samples from Senegal
}

\author{
Sokhna B Gueye ${ }^{1}$, Halimatou Diop-Ndiaye ${ }^{1}$, Aliou Gningue ${ }^{1}$, Ousseynou Ndiaye ${ }^{1}$, Abdou S Mbengue ${ }^{1}$, \\ Aïssatou Gaye-Diallo ${ }^{1}$, Angelique Ndjioyi ${ }^{2}$, Souleymane Mboup ${ }^{1}$, Coumba Touré-Kane ${ }^{1}$ \\ ${ }^{1}$ Laboratoire de Bactériologie-Virologie CHU Aristide Le Dantec, Université Cheikh Anta Diop, Dakar, Senegal \\ ${ }^{2}$ Laboratoire de microbiologie, Université des Sciences de la Santé, Libreville, Gabon
}

\begin{abstract}
Introduction: Chlamydia trachomatis and Neisseria gonorrhoeae are the most common causes of sexually transmitted disease in Senegal and worldwide. Molecular techniques have become the standard for their detection, and due to the frequency of co-infections, these tests can detect both agents and can be used on urine samples, vaginal swabs, or endocervical samples. In developing countries, the use of these molecular techniques is very limited and there is a need for evaluations of these techniques to be done.

Methodology: A total of 181 samples were tested with the Abbott RealTime CT/NG assay and compared with the Roche Cobas Amplicor $\mathrm{CT} / \mathrm{NG}$ assay. Specimens were collected from the key population of men having sex with men (urine, $\mathrm{n}=60$ ), female sex workers (genital swabs, $\mathrm{n}=60$ ) and from women visiting the laboratory for a gynecological checkup (urine, $\mathrm{n}=60$ and endocervical samples, $\mathrm{n}=61$ ).

Results: The agreement between the two techniques was $98.90 \%$ with a Kappa coefficient of 0.98 . A sensitivity of $93.3 \%$, a specificity of $100 \%$, a positive predictive value of $100 \%$, and a negative predictive value of $93.3 \%$ were found for both Chlamydia trachomatis and Neisseria gonorrhoeae.

Conclusion: These results showed that both methods are similar and suitable for the detection of CT/NG in all types of samples examined in this study.
\end{abstract}

Key words: Abbott RealTime CT/NG assay; urine; genital swabs.

J Infect Dev Ctries 2014; 8(7):898-903. doi:10.3855/jidc.4026

(Received 23 July 2013 - Accepted 08 February 2014)

Copyright (C) 2014 Gueye et al. This is an open-access article distributed under the Creative Commons Attribution License, which permits unrestricted use, distribution, and reproduction in any medium, provided the original work is properly cited.

\section{Introduction}

Chlamydia trachomatis (CT) and Neisseria gonorrhoeae (NG) are two of the most common bacterial causes of sexually transmitted infections (STIs) in the world [1], with a high incidence of new cases in developing countries [2,3]. These infections are often asymptomatic, especially in women. When untreated, they can lead to serious consequences such as pelvic inflammatory disease, ectopic pregnancy, and infertility $[4,5]$. Therefore, the early and accurate diagnosis of symptomatic and asymptomatic patients is essential for preventing complications and to control the spread of infection [6]. Currently, the benefits of diagnosis and the treatment options for these infections are well understood [7]. Since the advent of PCR testing, which is based on the detection and identification of specific DNA sequences, molecular techniques used for diagnosing $\mathrm{CT}$ and $\mathrm{NG}$ infections have become the best diagnostic methods in developed countries $[8,9]$.
Considering the frequency of co-infection, these tests have the advantage of detecting both agents [7,10-12] and can be used on both urine and endocervical samples [12-16]. In developing countries such as Senegal, the use of these molecular techniques is limited and there is a need to evaluate their performance.

The Abbott RealTime CT/NG assay is a real-time PCR method used for the direct qualitative detection of the plasmid DNA of Chlamydia trachomatis (CT) and the genomic DNA of Neisseria gonorrhoeae (NG) in endocervical or vaginal specimens, urethral samples, and urine samples from symptomatic or asymptomatic individuals. This technique was recently introduced into our laboratory and needed to be compared with our previously used platform, the Roche Cobas Amplicor CT/NG assay. Thus, this study evaluated the performance of the Abbott RealTime CT/NG assay for the detection of Chlamydia trachomatis and Neisseria gonorrhoeae in vaginal, endocervical, and urine samples. 


\section{Methodology}

Sample collection

This study examined a total of 181 samples, which included 120 urine samples, 48 self-vaginal swabs, and 13 endocervical swabs. The 120 urine samples were collected from 60 men who have sex with men (MSM) and from 60 consenting women who visited the laboratory for a gynecological checkup. The 13 endocervical swabs came from the same population of women, and the 48 vaginal swabs were collected from female sex workers (FSWs). The samples from the FSWs and MSM were collected during the National Survey of Combined Surveillance of STIs and HIV/AIDS (ENSC 2007) and the Epidemiological Survey of MSM in 2007, respectively. They were selected based on their positive results with the Roche Cobas Amplicor and were complemented by 60 negative samples. The endocervical specimens were transported in $1 \mathrm{~mL}$ of the in vitro specimen transport media (STM) and the urine samples were transported in sterile tubes without any additives. All specimens were stored at $-20^{\circ} \mathrm{C}$ until the time of testing. All samples were tested using two methods: the Roche Cobas Amplicor CT/NG assay and the Abbott RealTime CT/NG assay.

\section{Roche Cobas Amplicor CT/NG assay}

The Roche Cobas Amplicor CT/NG assay is a qualitative test performed in vitro for detecting the DNA of Chlamydia trachomatis and/or Neisseria gonorrhoeae in urogenital samples using the Cobas Amplicor automated analyzer. Additionally, this test allows for the detection of an internal control and can provide information about the presence of inhibitors. The target sequences of this test are the cryptic plasmid of Chlamydia trachomatis (207 base pairs) and the DNA methyl-transferase gene of Neisseria gonorrhoeae (201 base pairs). The assay consists of four steps: preparation of the sample, PCR amplification of the target DNA by complementary biotinylated primers, hybridization of amplicons with oligonucleotide probes, and detection of amplification products linked to the probe by a colorimetric determination.

Briefly, sample preparation begins with the cold lysis of $50 \mu \mathrm{L}$ of endocervical or vaginal fluid (in transport medium) or $500 \mu \mathrm{L}$ of urine using a chaotropic agent, followed by DNA precipitation with alcohol at $-70^{\circ} \mathrm{C}$. DNA amplification and detection were performed on the Cobas Amplicor analyzer as recommended by the manufacturer. The assay allows for the simultaneous amplification of the bacterial pathogen DNA (CT or NG) and the internal control (IC). A master mix contained a specific pair of biotinylated primers for the DNA of the bacterial pathogens and for the IC. The amplified DNA was detected using oligonucleotide probes specific to the target, which allowed for the independent identification of the bacterial pathogen amplicons and the IC. The results were interpreted using the optical density values (OD) of the sample and the IC, as measured at a $660 \mathrm{~nm}$ wavelength. The sample was considered negative if it had an OD of CT and/or NG $<0.2$ and an $\mathrm{OD}$ of $\mathrm{IC} \geq 0.2$. The sample was considered positive if it had an $\mathrm{OD}$ of $\mathrm{NG} \geq 0.2$ or an OD of $\mathrm{CT} \geq 0.8$ with any OD value of the IC. To confirm positive NG results, all positive samples were re-tested in duplicate.

When a sample had an OD of CT and/or NG that was $<0.2$ and an OD of IC that was $<0.2$, inhibitors were present in the sample and another aliquot was retested. Vaginal or endocervical samples that contained inhibitors were diluted by $1 / 10$ and retested after heating at $56^{\circ} \mathrm{C}$ for 10 minutes if the OD of IC remained $<0.2$. In contrast, urine samples that contained inhibitors were retested without treatment. If the OD of CT ranged between 0.2 and 0.8 with any value of the IC, the result was equivocal and the test was repeated in duplicate.

\section{Abbott RealTime CT/NG assay}

The Abbott RealTime CT/NG assay performed on an m2000 Abbott system is a qualitative test that includes the extraction of a DNA target in combination with an amplification-detection in real time. The assay targets a conserved region of the cryptic plasmid of $C$. trachomatis (102 base pairs) and a highly conserved region of the Opa gene of $N$. gonorrhoeae (122 base pairs).

The m2000 Abbott system consists of two instruments: the m2000sp, an automatic nucleic acid extractor, and the m2000rt, which ensures both amplification and detection in real time. Briefly, using the m2000sp instrument, $400 \mu \mathrm{L}$ of urine, endocervical or vaginal fluid were used for DNA extraction. The DNA extract obtained was added to a 96-well microplate before being mixed with the master mix. This plate was then sealed and manually transferred onto the m2000rt thermocycler, which ensured both the amplification and real-time detection of DNA using oligonucleotide probes that were labeled with fluorescence. The probes generated no signal unless specifically bound to the amplified product. The 
m2000rt automatically interpreted and provided the results.

One replicate of the negative control and two replicates of the cutoff positive control were required with each analysis. For each bacterial pathogen tested (CT or NG), the software calculated the mean target cycle number $(\mathrm{CN})$ using the control thresholds, and then added a predetermined number of cycles to this mean to generate the decision cycle threshold called the CO. If the tested sample generated a cycle number less than or equal to the $\mathrm{CO}$, the sample was denoted as positive and a numerical result higher than or equal to zero was reported. The numerical result (called delta cycle or DC) was the difference in cycles between the $\mathrm{CO}$ and the $\mathrm{CN}$ of the sample. When the numerical value of DC was high, the number of targets in the analyzed sample is more important. A sample denoted as negative in CT and a reported cycle number exceeding the decision cycle value were required for retesting. For negative samples that showed no amplification, the default value -1 was assigned to $\mathrm{CN}$ and DC. All tests were performed according to the manufacturer's instructions. Figures 1 and 2 present $\mathrm{CT}$ and NG real time PCR amplification curves.

\section{Analytical sensitivity}

According to the manufacturer, the limit of the detection claimed by the Abbott RealTime CT/NG assay is 320 copies of Chlamydia trachomatis (CT) target DNA and 320 copies of Neisseria gonorrhoeae (NG) target DNA per assay. The assay targets the Chlamydia trachomatis cryptic plasmid (present at

Figure 1. Chlamydia trachomatis (CT) amplification curves (internal control curves in grey; target curves in black) by Abbott RealTime CT/NG assay

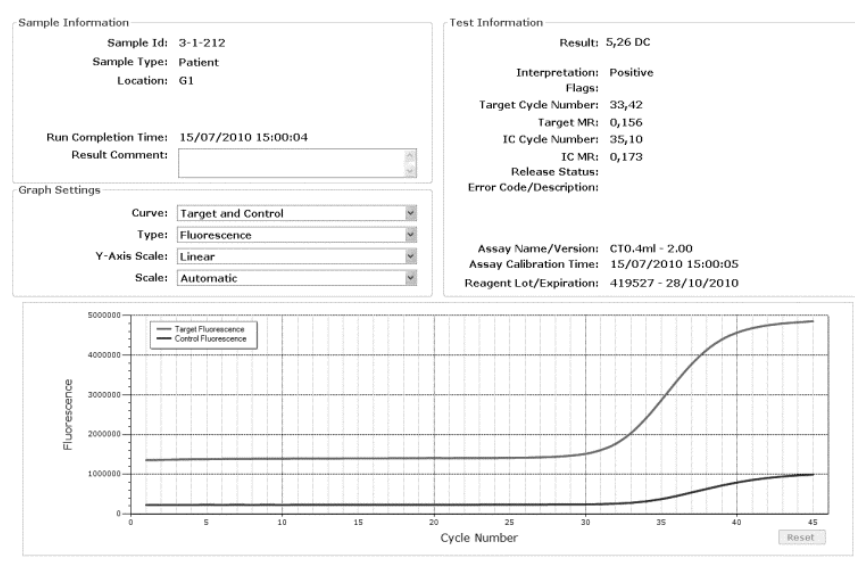

approximately 7 to 10 copies per Chlamydia organism) and the multicopy opacity gene of Neisseria gonorrhoeae (repeated up to 11 times per organism). Thus, 320 copies of target DNA are equivalent to approximately 30 to 40 organisms per assay.

\section{Statistical analysis}

To evaluate the performance of the Abbott RealTime CT/NG assay, the Roche Cobas Amplicor was used as the reference method. Statistical analysis was performed using the module StatCalc Epi Info, version 5.3. The confidence intervals were $95 \%$ and were calculated using a normal distribution. The diagnostic accuracy of the Abbott RealTime CT/NG assay was assessed using sensitivity (Se), specificity $(\mathrm{Sp})$, positive predictive value (PPV) (the number of true positives / [true positives + false positives]) and negative predictive value (NPV) (the number of true negatives / [true negatives + false negatives]). To determine the degree of concordance between the two techniques, a non-parametric Kappa test was used. Kappa allows for estimation and takes into account the chance of agreement or disagreement between qualitative judgments that are matched to detect and quantify the degree of agreement and to interpret the results. The possible Kappa values range from +1 for perfect agreement, 0 for the absence of agreement, and -1 in the case of full discordance [15].

Figure 2. Neisseria gonorrhoeae (NG) amplification curves (internal control curves in grey; target curves in black) by Abbott RealTime CT/NG assay

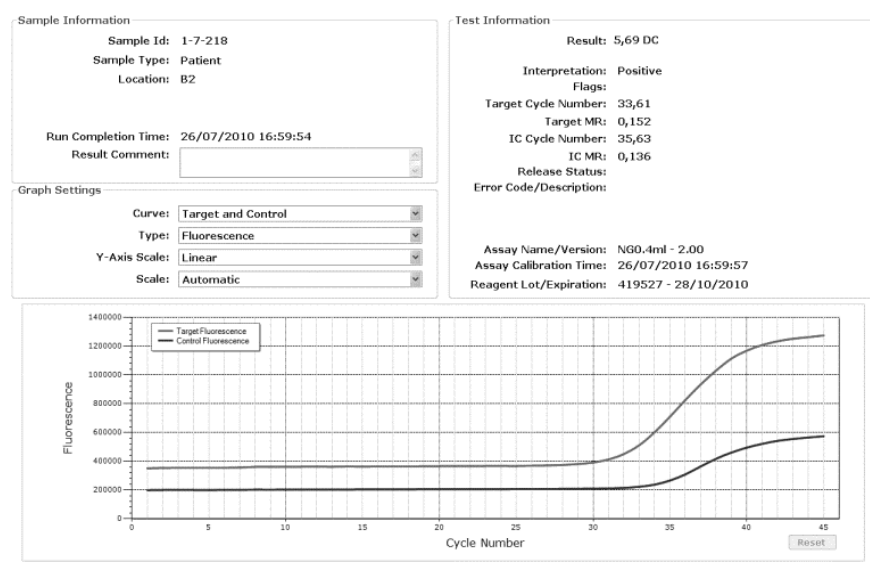




\section{Results}

Comparison of Cobas Amplicor and Abbott RealTime by target population and sample type

From the 181 samples tested with the Abbott RealTime and the Cobas Amplicor, two urine samples from the group of MSM were discordant: one for the detection of CT (MSM1181u) and another for the detection of NG (MSM1031u). This outcome gave an overall agreement percentage between the two assays of $99.5 \%(180 / 181)$ for each of the two bacterial agents. The two discordant samples were positive by the Cobas Amplicor assay and negative by the Abbott RealTime method, with a concordance percentage of $98.90 \%(179 / 181)$ between the two techniques. In summary, the Abbott RealTime assay identified 14 infections including 9 mono-infections of each agent CT and NG and 5 dual infections, while the Cobas Amplicor assay identified 15 infections - one additional mono-infection to those already detected by Abbott as showed in Table 1 and Table 2. Among these positive samples, 5 cases of co-infection with CT and NG were identified by each technique and these co-infections were from the same patients (4 MSM and 1 FSW) (Table 3).

In the samples collected from women visiting the laboratory ( $n=60$ urine and $n=13$ swabs), neither the Abbott RealTime nor the Cobas Amplicor assay detected an infection, representing an agreement of $100 \%$ between the two tests.

For the 48 self-vaginal swab samples from female sex workers, the two tests were in $100 \%$ agreement and showed 1 case $(2 \%)$ of co-infection with CT and $\mathrm{NG}$ and 2 cases $(4.16 \%)$ of isolated NG infection (Table 3). Therefore, the comparison between Abbott RealTime and Cobas Amplicor assays give almost identical results for each of these two bacterial pathogens with a Kappa coefficient of 1, a sensitivity of $100 \%$, a specificity of $100 \%$, a positive predictive value of $100 \%$, and a negative predictive value of $100 \%$.

For the 60 urine samples obtained from MSM, the two tests were in $96.66 \%$ agreement $(58 / 60)$ for both

Table 1. Contingency table of C. trachomatis in urine and vaginal samples

\begin{tabular}{|c|c|c|c|}
\hline \multirow{2}{*}{ Abbott RT } & \multicolumn{2}{|c|}{ Cobas Amplicor } & \multirow{2}{*}{ Total } \\
\hline & Positive & Negative & \\
\hline Negative & 1 & 166 & 167 \\
\hline
\end{tabular}

Sensibility: 93.3\% IC 95\% [64.2-99.6] ; Specificiy: 100\% IC 95\% [71.2-100] ; Positive predictive value: 100\% IC 95\% [71.7-100]; Negative predictive value : $93.3 \%$ IC $95 \%$ [66- 99.7]; Kappa coefficient : 0.98

Table 2. Contingency table of $N$. gonorrhoeae in urine and vaginal samples

\begin{tabular}{|c|c|c|c|}
\hline \multirow{2}{*}{ Abbott RT } & \multicolumn{2}{|c|}{ Cobas Amplicor } & \multirow{2}{*}{ Total } \\
\hline & Positive & Negative & \\
\hline Positive & 14 & 0 & 14 \\
\hline Negative & 1 & 166 & 167 \\
\hline
\end{tabular}

Sensibility: 93.3\% IC 95\% [64.2-99.6] ; Spécificity: 100\% IC 95\% [71.2-100] ; Positive predictive value: 100\% IC 95\% [71.7-100] ; Negative predictive value: $93.3 \%$ IC 95\% [66- 99.7]; Kappa coefficient : 0.98

Table 3. C. trachomatis and N. gonorrhoeae cases of infections and co-infections identified by Abbott RT and Cobas Amplicor

\begin{tabular}{ccccc}
\hline & Abbott RT & \multicolumn{2}{c}{ Cobas Amplicor } \\
& MSM & FSW & \multicolumn{2}{c}{$\begin{array}{c}\text { MSM } \\
(\mathbf{n}=\mathbf{6 0})\end{array}$} \\
\hline Isolated CT Infection & $(\mathbf{n = 6 0 )}$ & 0 & 10 & 0 \\
Isolated NG Infection & 7 & 2 & 8 & 2 \\
Co-infection & 4 & 1 & 4 & 1 \\
\hline
\end{tabular}

Table 4. Effect of inhibitors in invalid results

\begin{tabular}{ccc}
\hline & Cobas Amplicor & Abbott RT \\
\hline First test & 12 & 8 \\
Second test & 21 & 0 \\
Third test & 0 & NA \\
\hline
\end{tabular}

\footnotetext{
NA: not applicable
} 
CT and NG detection, with two discordant samples (positive by the Cobas Amplicor assay and negative by the Abbott RealTime assay). Indeed, the Abbott RealTime assay identified 9 cases of isolated CT infection, 7 cases of isolated NG infection, and 4 cases of co-infection with $\mathrm{CT}$ and $\mathrm{NG}$, while the Cobas Amplicor assay detected 10 cases of isolated CT infection, 8 cases of isolated NG infection, and 4 cases of co-infection with CT and NG (Table 3). Statistical analysis of the data yielded a Kappa coefficient of 0.98 for each bacterial agent.

\section{Performance of the Abbott RealTime assay}

An evaluation of the performance of the Abbott RealTime assay resulted in the following values for both CT and NG: sensitivity of $93.3 \%$ (CI 95\%: 64.299.6); specificity of $100 \%$ (CI 95\%: 71.2-100); PPV of $100 \%$ (CI 95\%: 71.7-100); and NPV of $93.3 \%$ (CI 95\% 66-99.7).

Furthermore, there were frequent instances of inhibitors in the tests using the Cobas Amplicor on endocervical samples. In self-vaginal swab samples from FSWs, 25\% (12/48) of the current results showed the presence of inhibitors after the first test, $44 \%$ $(21 / 48)$ after the second test, and the remainder underwent a third test. In contrast, with the m2000, $83 \%(40 / 48)$ of the results were effective after the first test and the presence of inhibitors was removed in the second test (Table 4).

\section{Discussion}

The positive CT and NG samples analyzed in this study were collected from epidemiological surveys conducted in 2007 among FSWs and MSM, who were considered high-risk groups, and the results from the Cobas Amplicor system had detected 25 positive samples in this data set. In fact, the incidence and prevalence of CT and NG infections are quite high in these groups because of their vulnerability to infection. In Senegal, the prevalences of CT and NG were $4 \%$ and $7.1 \%$ in registered FSWs [16] and $4.1 \%$ and 5.4\% among MSM [17], respectively. In contrast, neither $\mathrm{CT}$ nor NG was found in the samples of women who visited the laboratory. This may be due to the small sample size (60 patients) or the low prevalence of CT and $\mathrm{NG}$ in the general population $(0.3 \%$ and $0.2 \%$, respectively, in Senegal) [14]. The final results show excellent agreement between the results of the two assays for both $\mathrm{CT}$ and $\mathrm{NG}(\mathrm{K}=0.94$ for both bacterial pathogens).

For endocervical and self-vaginal swab samples, identical results were observed between the two assays after several re-tests of some samples; this may have been due to the presence of inhibitors. The similarity in the data may be related to our small sample size because discordances in data from larger data sets have previously been identified [10-12,18]. Indeed, an overall agreement of $98.5 \%$ between the results from the Abbott RealTime and Roche Cobas Amplicor assays in the diagnosis of CT on cervical swab samples was reported in $96.3 \%(26 / 27)$ of the positive results and in $100 \%(40 / 40)$ of the negative results [12]. Similarly, previous testing of endocervical swabs samples with the Abbott RealTime and Roche Cobas Amplicor assays showed an agreement of CT positivity of 98.8\% (confidence interval 95\%: 97.5\%$99.5 \%)$, CT negativity of $98.5 \%$ (96.8\% to $99.3 \%)$, NG positivity of $96.6 \%$ ( $88.3 \%$ to $99.1 \%)$, and NG negativity of $99.8 \%$ (99.2\% to $99.9 \%$ ) [10]. However, a discrepancy between the results of the two assays was observed for the detection of CT in 12 samples, with specifically six positive by the Cobas Amplicor assay and negative by the Abbott RealTime method that became negative after retesting with the Cobas Amplicor assay. All discrepant samples were confirmed to be negative by a third assay [10]. The low occurrence of inhibitors during analysis with the Abbott RealTime assay may be due to the additional heating step during the extraction, which helps to overcome this potential problem.

For the 120 urine samples, only two discrepancies were noted, all of which were in the MSM population. With the same type of sample, the Abbott RealTime assay already demonstrated an excellent ability to detect NG and CT with a sensitivity and specificity greater than $96 \%$ and $99 \%$ for $\mathrm{NG}$ and $92 \%$ and $99 \%$ for $\mathrm{CT}$, respectively, when compared with the $\mathrm{BD}$ Probe Tec ET and Aptima Combo 2 assay (AC2) $[7,11]$. Comparison with other assays, including the Roche Cobas Amplicor, also demonstrated the quality of the Abbott RealTime CT/NG assay for analyzing urine samples $[10,12]$.

\section{Conclusions}

The evaluation of the Abbott RealTime CT/NG assay for the qualitative detection of Chlamydia trachomatis and Neisseria gonorrhoeae in comparison with the Roche Cobas Amplicor showed good agreement between the results from the two assays, regardless of the type of sample. The Abbott RealTime assay is more specific and has a low rate of inhibition, which makes it more sensitive than the Cobas Amplicor assay, especially for vaginal and endocervical samples. In addition, the automation of 
DNA extraction, which reduces the number of manual steps and reduces the risk of contamination that is inherent in gene amplification techniques, is a suitable method to use in the diagnosis of Chlamydia trachomatis and Neisseria gonorrhoeae. However, the high cost of the nucleic acid extractor and reagents may limit its routine use, especially in countries with limited resources.

\section{References}

1. World Health Organization. 2001. Global Prevalence and Incidence of Selected Curable Sexually transmitted infections: Overview and Estimates. WHO, Geneva, Switzerland. Available at: http://www.who.int/hiv/pub/sti/who_hiv_aids_2001.02.pdf

2. Hammerschlag MR (2003) Implications of inappropriate sexually transmitted disease testing go beyond pure diagnostics. ASM News 69: 74-79.

3. Bébéar C, de Barbeyrac B (2009) Genital Chlamydia trachomatis infections. Clin Microbiol Infect 15: 4-10.

4. Peipert JF (2003) Genital chlamydial infections. N Engl J Med 349: 2424-2430.

5. Castellsagué X, Peeling RW, Franceschi S, de Sanjosé S, Smith JS, Albero G Díaz M, Herrero R, Muñoz N, Bosch FX; IARC Multicenter Cervical Cancer Study Group (2005) Chlamydia trachomatis infection in female partners of circumcised and uncircumcised adult men. Am J Epidemiol 162: 907-916.

6. Mangold KA, Regner MA, Tajuddin M, Tajuddin AM, Jennings L, Du H, Kaul KL (2007) Neisseria Species Identification Assay for the Confirmation of Neisseria gonorrhoeae-Positive Results of the COBAS Amplicor PCR. J Clin Microbiol 45: 1403-1409.

7. Levett PN, Brandt K, Olenius K, Brown C, Montgomery K, Horsman GB (2008) Evaluation of Three Automated Nucleic Acid Amplification Systems for the Detection of Chlamydia trachomatis and Neisseria gonorrhoeae in First-Void Urine Specimens. J Clin Microbiol 46: 2109-2111.

8. Garland SM, Tabrizi SN (2004) Diagnosis of sexually transmitted infections (STI) using self-collected non-invasive specimens. Sex Health 1: 121-126.

9. Bassiri M, Mårdh PA, Domeika M (1997) Multiplex AMPLICOR PCR screening for Chlamydia trachomatis and Neisseria gonorrhoeae in women attenting non-sexually transmitted disease clinics. The European Chlamydia Epidemiology Group. J Clin Microbiol 35: 2556-2560.

10. Cheng A, Qian Q, Kirby JE (2011) Evaluation of the Abbott RealTime CT/NG Assay in Comparison to the Roche Cobas Amplicor CT/NG Assay. J Clin Microbiol 49: 1294-1300.
11. Gaydos CA, Cartwright CP, Colaninno P, Welsch J, Holden J, Ho SY, Webb EM, Anderson C, Bertuzis R, Zhang L, Miller T, Leckie G, Abravaya K, Robinson J (2010) Performance of the Abbott RealTime CT/NG for Detection of Chlamydia trachomatis and Neisseria gonorrhoeae. J Clin Microbiol 48: 3236-3243.

12. Marshall R, Chernesky M, Jang D, Hook EW, Cartwright CP, Howell-Adams B, Ho S, Welk J, Lai-Zhang J, Brashear J, Diedrich B, Otis K, Webb E, Robinson J, Yu H (2007) Characteristics of the m2000 Automated Sample Preparation and Multiplex Real-Time PCR System for Detection of Chlamydia trachomatis and Neisseria gonorrhoeae. J Clin Microbiol 45: 747-751.

13. Lowe P, O'Loughlin P, Evans K, White M, Bartley PB, Vohra R (2006) Comparison of the Gen-Probe APTIMA Combo 2 Assay to the AMPLICOR CT/NG Assay for Detection of Chlamydia trachomatis and Neisseria gonorrhoeae in Urine Samples from Australian Men and Women. J Clin Microbiol 44: 2619-2621.

14. Gueye Ndiaye A, Faye CM, Ndiaye I, Fall K, Gueye Gaye A, Diop IL, Mboup S (2009) Screening for HIV, syphilis, Chlamydia trachomatis and Neisseria gonorrhoreae during a combined survey conducted in Malicouna, a Senegalese rural area. Bull Soc Pathol Exot 102: 150-154.

15. Bergeri I, Michel R, Boutin J-B (2002) Pour tout savoir ou presque sur le coefficient Kappa. Med Trop 62: 634-636.

16. Laurent C, Seck K, Coumba N, Kane T, Samb N, Wade A Liégeois F, Mboup S, Ndoye I, Delaporte E (2003) Prevalence of HIV and other sexually transmitted infections, and risk behaviours in unregistered sex workers in Dakar, Senegal. AIDS 17: 1811-186.

17. Wade AS, Kane CT, Diallo PA, Diop AK, Gueye K, Mboup S Ndoye I, Lagarde E (2005) HIV infection and sexally transmitted infections among men who have sex with men in Senegal. AIDS 19: 2133-2140.

18. Chernesky M, Jang D, Portillo E, Smieja M, Kapala J, Doucette C Sumner J, Ewert R, MacRitchie C, Gilchrist J (2012) Comparison of three assays for detection of Chlamydia trachomatis and Neisseria gonorrhoeae in SurePath Pap samples and the role of pre- and postcytology testing. J Clin Microbiol 50: 1281-1284.

\section{Corresponding author}

Dr. Halimatou Diop-Ndiaye

Université Cheikh Anta Diop, Dakar and Laboratoire de

Bactériologie-virologie

CHU Aristide Le Dantec, Dakar, Sénégal

Phone: (221) 338216420

Fax: (221) 338216442

Email: halimatoudiop@yahoo.fr

Conflict of interests: No conflict of interests is declared. 